\title{
The relationship between fertility and lifespan in humans
}

\author{
Maris Kuningas • Signe Altmäe • \\ André G. Uitterlinden • Albert Hofman • \\ Cornelia M. van Duijn • Henning Tiemeier
}

Received: 27 September 2010 / Accepted: 13 December 2010/Published online: 11 January 2011

(C) The Author(s) 2011. This article is published with open access at Springerlink.com

\begin{abstract}
Evolutionary theories of aging predict a trade-off between fertility and lifespan, where increased lifespan comes at the cost of reduced fertility. Support for this prediction has been obtained from
\end{abstract}

Electronic supplementary material The online version of this article (doi:10.1007/s11357-010-9202-4) contains

supplementary material, which is available to authorized users.

M. Kuningas $(\bowtie) \cdot$ A. Hofman · C. M. van Duijn •

H. Tiemeier

Department of Epidemiology,

Erasmus University Medical Center,

P.O. Box 20403000 CA, Rotterdam, The Netherlands

e-mail: M.Kuningas@erasmusmc.nl

S. Altmäe

Department of Clinical Science, Intervention

and Technology, Division of Obstetrics and Gynaecology,

Karolinska Institutet,

Karolinska University Hospital Huddinge,

Stockholm, Sweden

S. Altmäe

Competence Centre on Reproductive Medicine and Biology,

Tartu, Estonia

A. G. Uitterlinden

Department of Internal Medicine,

Erasmus University Medical Center,

Rotterdam, The Netherlands

\section{H. Tiemeier}

Department of Child and Adolescent Psychiatry, Erasmus Medical Center-Sophia Children's Hospital, Rotterdam, The Netherlands various sources. However, which genes underlie this relationship is unknown. To assess it, we first analyzed the association of fertility with age at menarche and menopause, and with mortality in 3,575 married female participants of the Rotterdam Study. In addition, we conducted a candidate gene study where 1,664 single nucleotide polymorphisms (SNPs) in 25 candidate genes were analyzed in relation to number of children as a measure of fertility. SNPs that associated with fertility were analyzed for association with mortality. We observed no associations between fertility and age at menarche $(p=0.38)$ and menopause $(p=0.07)$. In contrast, fertility was associated with mortality. Women with two to three children had significantly lower mortality (hazard ratio (HR), 0.82; 95\% confidence interval (95\% CI), 0.69-0.97) compared to women with no children. No such benefit was observed for women with four or more children, who had a similar mortality risk (HR, 0.93; 95\% CI, 0.76-1.13) as women with no children. The analysis of candidate genes revealed four genes that influence fertility after correction for multiple testing: $C G B / L H B$ gene cluster $(p=0.0036), F S H R(p=0.023), F S T(p=0.023)$, and INHBA $(p=0.021)$. However, none of the independent SNPs in these genes predicted mortality. In conclusion, women who bear two to three children live longer than those who bear none or many children, but this relationship was not mediated by the candidate genes analyzed in this study. 
Keywords Fertility $\cdot$ Lifespan · Trade-off · Gene $\cdot$ SNP

\section{Introduction}

Evolutionary theories of aging predict a trade-off between fertility and lifespan, where increased lifespan comes at the cost of reduced fertility (Kirkwood 1977; Williams 1957). Studies in model organisms have provided evidence for this prediction (Rose and Charlesworth 1980; Stearns and Partridge 2001). In humans, however, no convincing support for this hypothesis has been obtained when historical populations were studied. However, in contemporary populations, a trade-off between fertility and lifespan has been observed (Hurt et al. 2006; Le Bourg 2007). It is likely that genes underlie this relationship but the identity of these genes has largely remained unknown.

According to the antagonistic pleiotropy theory (Williams 1957) and the disposable soma theory (Kirkwood and Rose 1991; Kirkwood 1977) of aging, a trade-off between reproduction and lifespan exists. The disposable soma theory emphasizes energetic and metabolic costs associated with reproduction, which may lead to deterioration in maternal condition, increase risk of disease, and lead to higher mortality. The antagonistic pleiotropy theory, on the other hand, emphasizes a genetic trade-off, where genes that increase reproductive potential early in life increase risk of disease and mortality later in life. Such a tradeoff would be expected even for women who have not given birth but who carry these gene variants. Several researchers have sought evidence for the trade-off between fertility and lifespan, with elusive results. In historical populations, negative (Korpelainen 2000; Penn and Smith 2007; Westendorp and Kirkwood 1998), positive (Korpelainen 2000; Le Bourg 2007; McArdle et al. 2006; Muller et al. 2002), and no relationship (Helle et al. 2004; Lycett et al. 2000) between fertility and lifespan have been reported. In contemporary populations, however, the results are more uniform. In several studies, it has been observed that women with no children and women with more than four children have the highest mortality (Doblhammer 2000; Hurt et al. 2006)

The increased mortality for women with no or with many children suggests that either the same or different genes are at play. To date, a number of genes have been identified to influence female fertility. In addition, it has been estimated that the heritability of female lifetime reproductive success in a contemporary population is $22 \%$ (Kosova et al. 2010) whereas in a preindustrial population, it was estimated to reach $47 \%$ (Pettay et al. 2005). These estimates reflect an underlying genetic architecture of female fertility. According to the antagonistic pleiotropy theory of aging, the same genes should influence human lifespan.

Evidence suggests that age at menarche and menopause also influence female fertility. It has been found that women with earlier menarche have higher fertility (Borgerhoff Mulder 1989), but not all studies corroborate this finding (Helm et al. 1995; Sandler et al. 1984). Likewise, later age at menopause has been related to higher number of live births (Bromberger et al. 1997; Parazzini et al. 1992; Stanford et al. 1987; Whelan et al. 1990). In addition, later age at menopause has been associated with increased risk of breast and endometrial cancer, but with decreased risk of osteoporosis and cardiovascular diseases $(\mathrm{Hu}$ et al. 1999; Sowers and La Pietra 1995). These effects have been attributed to high lifetime exposure to estrogens. Given these associations, it is likely that age at menarche and at menopause could predict mortality at old age.

In this study, we set out to examine the relationship between fertility and lifespan and explore the influences of age at menarche and menopause on these phenotypes. In addition, we conducted a candidate gene study in order to identify genes that could influence both fertility and lifespan in an antagonistic manner. We expected that genetic variants that associate with increased fertility would result in higher mortality. The study was carried out in married female participants of the prospective Rotterdam Study.

\section{Methods}

Study population

This study is embedded in the Rotterdam Study, an ongoing population-based cohort on risk factors for chronic diseases in the elderly. Detailed information on design, objectives, and methods has been presented elsewhere (Hofman et al. 2009). For this study, data from the first (RSI) and second (RSII) cohort of 
the Rotterdam Study were available. In RSI, all inhabitants aged over 55 years living in the Ommoord district of Rotterdam were invited to participate. Of these 7,983 (78\%) agreed to participate. In 1999, 3,011 participants (out of 4,472 invitees) who had become 55 years of age or moved into the study district since the start of the study were added to the cohort (RSII). All participants of the Rotterdam Study were followed for mortality until January 1, 2009. The current study included 3,575 married female participants of the Rotterdam Study. The Medical Ethics Committee of the Erasmus Medical Center approved the Rotterdam Study, and written informed consent was obtained from all participants.

Fertility and age at menarche and at menopause

As a measure of fertility, data on self-reported number of children were used. Women were asked, "How many children do you have?" Fertility was analyzed linearly but also categorically, where women were categorized into the following groups: (1) childless vs. with children and (2) no children, one child, two to three children, and four or more children. Data on age at menarche were collected by asking women, "How old were you when you had your first menstrual period?", and data on age at menopause were collected by asking women, "How old were you when you had your last menstrual period?" Selfreported age at menopause was defined as 12 months after periods had ceased. The retrospective data on self-reported number of children, age at menarche and at menopause were collected by a questionnaire during the first RSI interview (1990-1993) and during the first RSII interview (1999-2001).

\section{Confounders}

The following variables were considered as possible confounders: age at baseline, date of birth, smoking, and socioeconomic status. Smoking status was defined as having never smoked, being former or being current smoker. To correct for differences in socioeconomic status, data on education and household income were used. The participants were asked about their formal education, the number of years in each type of education, and whether education had been completed. Based on these data, education was divided into two levels: lower and higher education.
The lower education category included participants with primary, lower vocational, and lower secondary education. The high education category included participants with intermediate vocational, general secondary, higher vocational, and university education. Household income was classified into 13 precoded categories. Equivalent household income was defined as the midpoint of each income category divided by the number of persons living on that income raised to the power 0.36 (Buhmann et al. 1988).

Candidate genes and genotype data

Altogether, 25 candidate genes were selected based on their involvement in female fertility according to the published literature (Supplementary table 1). These genes function in hypothalamic-pituitary-ovarian (HPO) axis, folliculogenesis, and implantation processes. From these genes and within $10-\mathrm{kb}$ vicinity 1,756 single nucleotide polymorphisms (SNPs) were selected for study. Genotype data for these SNPs were obtained from genome-wide genotyping data of the Rotterdam Study. The genome-wide genotyping was performed with Illumina $550 \mathrm{~K}$ array (Illumina, San Diego, CA, USA) in self-reported Caucasian individuals (sample call rate, $\geq 97.5 \%$ ). Individuals with excess of autosomal heterozygosity, mismatch between genotypic and phenotypic gender, and outliers identified by the identity-by-state clustering analysis were excluded. This genotype data were used to impute 2.5 million autosomal SNPs described in HapMap's Phase II European population panel with the imputation software MACH (http://www.sph. umich.edu/csg/abecasis/MACH). Such SNP imputation infers genotypes probabilistically according to shared haplotype stretches between the study samples and HapMap release 22 build 36 data. From the 1,756 SNPs in the candidate genes, 62 were excluded due to a minor allele frequency (MAF) of less than $1 \%$ and 30 SNPs due to the Hardy-Weinberg equilibrium $p$ value lower than $1 \%$, resulting in 1,664 SNPs for further analyses.

Statistical analysis

First, we analyzed the association between fertility and age at menarche and age at menopause with linear regression. Second, we assessed the relation of 
fertility, age at menarche and at menopause with mortality using Cox proportional hazard regression. Third, the function- and gene-wide influence of SNPs on fertility was determined with a set-based test, implemented in PLINK (Purcell et al. 2007) (http:// pngu.mgh.harvard.edu/ purcell/plink/). The default settings of PLINK were used $\left(r^{2}=0.05 ; p\right.$ value $<$ 0.05 ; max number of SNPs, 5). The significance of SNP combinations within a function or a gene was estimated using 10,000 permutations. Where a function or a gene met a significance threshold of $p<0.05$ after permutations, independently associated SNPs within these, as defined by PLINK, were analyzed further for association with fertility and mortality. Fourth, the association between SNPs and fertility was assessed with logistic regression. Finally, the association between SNPs and mortality was analyzed with Cox proportional hazard regression. All analyses were adjusted for birth year and education, except the mortality analyses which were additionally adjusted for age at baseline and education. All analyses were repeated with additional adjustment for household income and smoking status. Data were analyzed with SPSS version 17 (SPSS Inc, Chicago, IL, USA) and PLINK version 1.07 (Purcell et al. 2007) statistical software.

\section{Results}

The characteristics of the 3,575 study participants are presented in Table 1. All women were born between 1893 and 1944, and their mean age at baseline was

Table 1 Characteristics of the study participants

\begin{tabular}{lr}
\hline Number & 3,575 \\
Age at baseline (mean, SD) & $68(9.49)$ \\
Low education $(n, \%)$ & $2,370(67 \%)$ \\
Age at menarche (mean, SD) & $13.6(1.78)$ \\
Age at menopause (mean, SD) & $48.8(5.31)$ \\
Fertility & \\
0 children $(n, \%)$ & $471(13 \%)$ \\
1 child $(n, \%)$ & $577(16 \%)$ \\
$2-3$ children $(n, \%)$ & $1,975(55 \%)$ \\
$\geq 4$ children $(n, \%)$ & $552(16 \%)$ \\
Mortality $(n, \%)$ & $1,116 / 3,238(66 \%)$ \\
Follow-up (mean, SD) & $12.1(4.88)$ \\
\hline
\end{tabular}

68 years. Of the 3,575 participants, 2,370 (67\%) had a lower education. The number of children in this population ranged from 0 to 16 (mean (SD), 2.22 $(1.53))$. Women with no children $(n=471)$ constituted $13 \%$ of the population. Data on mortality were available for 3,238 participants. Of these participants, $1,116(66 \%)$ died during a mean follow-up period of 12.1 years.

In this study, age at menarche and at menopause had no pronounced influence on fertility, neither when analyzed linearly nor when categorizing women based on the number of children they have had (Table 2). The only trend observed involved women with two or three children who had later menopause compared to women with no children (estimate standard error (SE), $0.56(0.29), p=0.06)$. This trend was also reflected when fertility was analyzed by comparing women who have had children with women with no children (estimate (SE), 0.49 (0.28), $p=0.07$ ). In contrast, mortality risks were not influenced by age at menarche (hazard ratio (HR), $0.99 ; 95 \%$ confidence interval (95\% CI), 0.95-1.02) and at menopause (HR, 0.99; 95\% CI, 0.98-1.00; Table 2).

Next, we assessed the association between fertility and all-cause mortality. We observed no linear relation between the number of children and mortality (HR, 0.99; 95\% CI, 0.95-1.02; Table 2). But, when comparing women with two or three children with women with no children, it became apparent that they have significantly lower mortality (HR, $0.82 ; 95 \% \mathrm{CI}$, 0.69-0.97; Fig. 1). No such benefit was observed for women with four or more children, who had a similar mortality risk (HR, 0.93; 95\% CI, 0.76-1.13) as women with no children (Table 2), but a higher mortality risk when compared to women with two to three children (HR, 1.13; 95\% CI, 0.96-1.32).

To explore the influence of genetic variation on fertility, we first assessed the combined effect of SNPs in folliculogenesis, HPO axis, and implantation on fertility. From these three functions, the combined effect of SNPs involved in HPO axis revealed a trend for association with fertility after permutations $(p=$ 0.09; Supplementary table 2). To determine which of the 25 selected candidate genes contain SNPs that influence fertility the most, we performed a gene-wide association analysis. This approach revealed four genes that influence fertility after permutations: $C G B / L H B(0$ vs. $2-3$ children, $p=0.0036), F S H R$ ( 0 vs. $\geq 4$ children, $p=0.023), F S T$ ( 0 vs. $\geq 4$ children, $p=0.023$ ), and 
Table 2 Association of fertility with age at menarche and menopause, and with mortality

The number of deaths during follow-up in the different fertility categories: 0 children $(n=189)$, one child $(n=201)$, 2-3 children $(n=512)$, $\geq 4$ children $(n=214)$

$* p<0.05$

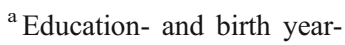
adjusted linear regression

${ }^{\mathrm{b}} \mathrm{Cox}$ proportional hazard model adjusted for education and age at baseline

\begin{tabular}{|c|c|c|c|c|c|}
\hline & \multicolumn{2}{|l|}{ Menarche $^{\mathrm{a}}$} & \multicolumn{2}{|l|}{ Menopause $^{\mathrm{a}}$} & \multirow{2}{*}{$\begin{array}{l}\text { Mortality }^{\mathrm{b}} \\
\text { HR }(95 \% \mathrm{CI})\end{array}$} \\
\hline & Estimate (SE) & $p$ value & Estimate (SE) & $p$ value & \\
\hline \multicolumn{6}{|l|}{ Fertility } \\
\hline 0 children & Reference & - & Reference & - & Reference \\
\hline 1 child & $-0.18(0.11)$ & 0.10 & $0.31(0.34)$ & 0.37 & $0.96(0.79-1.17)$ \\
\hline 2-3 children & $-0.11(0.09)$ & 0.25 & $0.56(0.29)$ & 0.06 & $0.82(0.69-0.97)^{*}$ \\
\hline$\geq 4$ children & $0.13(0.11)$ & 0.27 & $0.49(0.35)$ & 0.16 & $0.93(0.76-1.13)$ \\
\hline Fertile (yes/no) & $-0.08(0.09)$ & 0.38 & $0.49(0.28)$ & 0.07 & $0.87(0.74-1.02)$ \\
\hline Per child & $0.02(0.02)$ & 0.21 & $0.10(0.06)$ & 0.09 & $0.99(0.95-1.02)$ \\
\hline Age at menarche & - & - & - & - & $0.99(0.95-1.02)$ \\
\hline Age at menopause & - & - & - & - & $0.99(0.98-1.00)$ \\
\hline
\end{tabular}

INHBA (0 vs. 1 child, $p=0.021$ ) (Supplementary table 2). Independent SNPs from these four candidate genes were further examined for their association with fertility and mortality.

The $C G B / L H B$ gene cluster contained three SNPs, and FSHR contained five SNPs whereas FST and INHBA both contained one independent SNP. For all analyzed SNPs, a similar influence across the fertility categories was observed (Table 3). For instance, women carrying the rs1062708 SNP in CGB/LHB gene cluster had a high probability to have one child (OR, 1.20; 95\% CI, 1.01-1.45), but lower probabilities to have two to three (OR, 1.13; 95\% CI, 0.97-1.31) or four or more children (OR, 1.07; 95\% CI, 0.89-1.27) when compared to women with no children. Finally, we tested the association of these SNPs with mortality. Despite their association with fertility, none of these SNPs directly predicted mortality (see also Table 3 ).

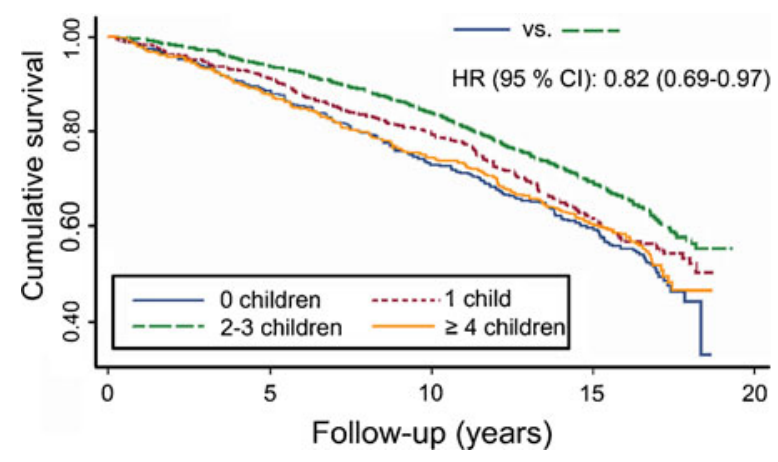

Fig. 1 Kaplan-Meier survival curves for women with different numbers of children. The hazard ratio (HR) with $95 \%$ confidence interval (CI) for the comparison of women with no children and women with two to three children is adjusted for age at baseline and education
All reported analyses were repeated with additional adjustment for household income and smoking status, without leading to changes in results (data not shown).

\section{Discussion}

In this study, we found that women with two or three children have lower mortality compared to women with no children. No such benefit was observed for women with four or more children, who had a similar mortality risk as women with no children. This relationship between fertility and lifespan was not mediated by the analyzed candidate genes. In addition, we found no pronounced influence of age at menarche and at menopause on fertility and lifespan.

The relationship between fertility and lifespan has previously been studied by a number of studies. Similar to other studies (Hurt et al. 2006), we found no linear relationship between number of children and lifespan. Instead, we observed that women with no children and with four or more children had the highest mortality. It has been hypothesized that with repeated pregnancies, women become depleted of resources that would otherwise be available for maintenance and repair of the body, and hence, lead to higher mortality (Kirkwood and Rose 1991; Winkvist et al. 1992). In addition, it has been suggested that repeated pregnancies result in permanent detrimental effects on lipid and glucose metabolism (Martin et al. 1999; Sattar and Greer 2002) and this way contribute to higher risk for coronary artery disease, stroke, diabetes (Lawlor et al. 2003; Qureshi 
Table 3 Influence of SNPs in candidate genes on fertility and mortality

\begin{tabular}{|c|c|c|c|c|c|}
\hline \multirow[b]{2}{*}{ Gene } & \multirow[b]{2}{*}{ MAF } & \multicolumn{3}{|l|}{ Fertility } & \multirow{2}{*}{$\begin{array}{l}\text { Mortality } \\
\text { HR (95\% CI) }\end{array}$} \\
\hline & & $\begin{array}{l}0 \text { vs. } 1 \text { child } \\
\text { OR }(95 \% \text { CI })\end{array}$ & $\begin{array}{l}0 \text { vs. } 2-3 \text { children } \\
\text { OR }(95 \% \mathrm{CI})\end{array}$ & $\begin{array}{l}0 \text { vs. } \geq 4 \text { children } \\
\text { OR }(95 \% \mathrm{CI})\end{array}$ & \\
\hline \multicolumn{6}{|l|}{$C G B / L H B$} \\
\hline rs753308 & 0.41 & $0.70(0.59-0.86)^{* *}$ & $0.78(0.67-0.91)^{* *}$ & $0.74(0.62-0.89)^{* *}$ & $1.01(0.93-1.10)$ \\
\hline rs 3752210 & 0.41 & $0.70(0.61-0.89)^{* *}$ & $0.78(0.67-0.90)^{* *}$ & $0.74(0.62-0.89)^{* *}$ & $1.03(0.94-1.11)$ \\
\hline rs1062708 & 0.49 & $1.20(1.01-1.45)^{*}$ & $1.13(0.97-1.31)$ & $1.07(0.89-1.27)$ & $1.02(0.94-1.11)$ \\
\hline \multicolumn{6}{|l|}{ FSHR } \\
\hline rs1277460 & 0.21 & $0.90(0.70-1.07)$ & $0.89(0.75-1.06)$ & $0.66(0.52-0.83)^{* *}$ & $0.95(0.85-1.05)$ \\
\hline rs6545094 & 0.42 & $1.20(1.03-1.50)^{*}$ & $1.20(1.03-1.40)^{*}$ & $1.35(1.11-1.64)^{* *}$ & $0.98(0.90-1.06)$ \\
\hline rs3913665 & 0.41 & $0.90(0.71-1.02)$ & $0.92(0.79-1.07)$ & $0.75(0.62-0.91)^{* *}$ & $1.03(0.94-1.12)$ \\
\hline rs971831 & 0.30 & $1.10(0.90-1.34)$ & $1.12(0.95-1.32)$ & $1.31(1.06-1.60)^{*}$ & $1.00(0.92-1.10)$ \\
\hline rs2268360 & 0.22 & $0.90(0.75-1.16)$ & $0.90(0.76-1.07)$ & $0.76(0.61-0.94)^{*}$ & $0.98(0.89-1.08)$ \\
\hline \multicolumn{6}{|l|}{$F S T$} \\
\hline rs 10080213 & 0.24 & $1.30(1.03-1.58)^{*}$ & $1.15(0.96-1.38)$ & $1.37(1.10-1.70)^{* *}$ & $1.01(0.92-1.12)$ \\
\hline \multicolumn{6}{|l|}{$I N H B A$} \\
\hline rs 2237432 & 0.25 & $1.37(1.10-1.71)^{* *}$ & $1.24(1.04-1.49)^{*}$ & $1.11(0.89-1.38)$ & $0.97(0.88-1.07)$ \\
\hline
\end{tabular}

Association with fertility was analyzed with birth year- and education-adjusted logistic regression; association with mortality was analyzed with age at baseline and education-adjusted Cox proportional hazard model

$M A F$ minor allele frequency, $O R$ odds ratio, $H R$ hazard ratio, $C I$ confidence interval

${ }^{*} p<0.05 ; * * p<0.005$

et al. 1997), and to higher mortality later in life. On the other hand, the higher mortality among women with no children has probably different biological causes. It is likely that mechanisms that lead to infertility have also detrimental effects on other physiological processes, thereby contributing to higher mortality.

Female reproductive lifespan is determined by the onset and cessation of menstruation. It has been found that both the age at menarche and at menopause influence fertility (Borgerhoff Mulder 1989; Parazzini et al. 1992; Stanford et al. 1987; Whelan et al. 1990). In this study, we found no effects of age at menarche on fertility. For age at menopause, in contrast, we observed that women with children tended to have their menopause at later ages than women without children. Interestingly, it has been reported that women who have their menopause at later ages have decreased risk of having a heart attack, stroke, or other cardiovascular disease event, but increased risk of having breast or endometrial cancer ( $\mathrm{Hu}$ et al. 1999; Sowers and La Pietra 1995). These associations are in an opposite direction than the disease risks associated with higher fertility, which highlight the complex interactions between them. This might also explain why no association between age at menopause and mortality was observed in this study.

Over the years, a number of genes have been identified to influence fertility. In this study, we selected candidate genes that are involved in HPO axis, folliculogenesis, and implantation process. From these, three genes $(C G B / L H B$ gene cluster, FSHR, and $I N H B A)$ involved in HPO axis and one gene (FST) involved in folliculogenesis were associated with fertility. The $C G B / L H B$ gene cluster is located in a gene-rich region which is targeted by natural selection and gene conversion events (Nagirnaja et al. 2010). Given the association of the $C G B / L H B$ gene cluster with fertility and the fact that it is located in a region under natural selection, an influence of genetic variants in this gene cluster on lifespan would have been expected. This, however, was not the case. Likewise, an effect on mortality would have been expected for the FSHR gene variants since SNPs in FSHR have been associated with hypertension (Nakayama et al. 2006), bone mineral density 
(Rendina et al. 2010), and ovarian cancer (Yang et al. 2006). In this study, however, genetic variants in FSHR gene influenced only fertility and not mortality, suggesting that the effect of FSHR gene variants on the other phenotypes is not fatal.

In exploring the relationship between fertility and lifespan, we selected candidate genes that have been shown to be involved in fertility. Another approach would be to select genes that have been associated with lifespan and assess their influence on fertility. This approach was recently applied in a study analyzing the influence of $A C E, P O N 1, P P A R G$, and $A P O E$ genes on fertility in 151 healthy unrelated subjects in post-reproductive age (Corbo et al. 2008). It was found that $A P O E^{*} 2$ allele results in a trade-off with fertility whereas the PPARG Pro/Ala genotype contributes to beneficial effects both in early and in late life in a gender-specific way. These results warrant further research in analyzing candidate longevity genes in relation to fertility.

A strength of the current study is the large study population with a long follow-up time for mortality. In addition, the availability of genome-wide genetic data enabled us to investigate all to date known common genetic variants in the selected candidate genes. Another strength includes the fact that a contemporary population was analyzed, which is less selected than those used in historical studies (Hurt et al. 2006). Furthermore, in contemporary populations, data on birth and death dates and on the number of children are likely to be more accurate than for historical populations. On the other hand, the use of a contemporary population poses also a problem due to the use of birth control. Therefore, the reported fertility would not entirely reflect natural fertility. However, participants of this population were fertile before the use of oral contraceptives became widespread. In addition, we were able to adjust for the year of birth, which should account for the differences in fertility patterns over the years. A more likely source of bias would be the self-reported age at menarche and at menopause since older women may not accurately recall these ages.

In conclusion, in this study, we observed that women who bear fewer children are predicted to live longer than those who bear none or many children. In addition, we identified a number of SNPs that associate with fertility. Despite that, none of the identified SNPs associated with mortality. In this study, we selected candidate genes based on their direct role in HPO axis, folliculogenesis, and implantation. It might be that genes influencing fertility indirectly, either through lipid or glucose metabolism, innate immunity, nutrition, or stress, are more likely candidates to affect human lifespan.

Acknowledgements This study was supported by the Netherlands Organization of Scientific Research (grant no 175.010.2005.011); the Netherlands Genomics Initiative (NGI)/Netherlands Organization for Scientific Research (NWO); Netherlands Consortium for Healthy Ageing (grant no 050-060-810); the Erasmus Medical Center, and Erasmus University in Rotterdam; the Netherlands Organization for Health Research and Development; the Netherlands Research Institute for Diseases in the Elderly; the Dutch Ministry of Education, Culture and Science, and the Ministry for Health, Welfare and Sports; the European Commission; and the Municipality of Rotterdam, the Netherlands. H.T was supported by Netherlands Organisation for Scientific Research (grant no VIDI 017-106-370) and S.A. by Karolinska Institutet and Enterprise Estonia (grant no EU30200). The funders had no role in the study design or data collection and analysis. We would like to thank everyone involved in the data collection process of the Rotterdam study and the inhabitants of Ommoord for their time and effort.

Competing interests The authors declare that there are no competing interests associated with this study.

Open Access This article is distributed under the terms of the Creative Commons Attribution Noncommercial License which permits any noncommercial use, distribution, and reproduction in any medium, provided the original author(s) and source are credited.

\section{References}

Borgerhoff Mulder M (1989) Menarche, menopause and reproduction in the Kipsigis of Kenya. J Biosoc Sci 21:179-192

Bromberger JT, Matthews KA, Kuller LH, Wing RR, Meilahn EN, Plantinga P (1997) Prospective study of the determinants of age at menopause. Am J Epidemiol 145:124-133

Buhmann B, Rainwater L, Schmaus G, Smeeding T (1988) Equivalence scales well-being, inequality and poverty: sensitivity estimates across ten countries using the Luxembourg Income Study (LIS) data base. Rev Income Wealth $34: 115-142$

Corbo RM, Ulizzi L, Piombo L, Scacchi R (2008) Study on a possible effect of four longevity candidate genes (ACE, PON1, PPAR-gamma, and APOE) on human fertility. Biogerontology 9:317-323

Doblhammer G (2000) Reproductive history and mortality later in life: a comparative study of England and Wales and Austria. Popul Stud Camb 54:169-176 
Helle S, Lummaa V, Jokela J (2004) Accelerated immunosenescence in preindustrial twin mothers. Proc Natl Acad Sci USA 101:12391-12396

Helm P, Munster K, Schmidt L (1995) Recalled menarche in relation to infertility and adult weight and height. Acta Obstet Gynecol Scand 74:718-722

Hofman A, Breteler MM, van Duijn CM, Janssen HL, Krestin GP, Kuipers EJ, Stricker BH, Tiemeier H, Uitterlinden AG, Vingerling JR, Witteman JC (2009) The Rotterdam Study: 2010 objectives and design update. Eur J Epidemiol 24:553-572

Hu FB, Grodstein F, Hennekens CH, Colditz GA, Johnson M, Manson JE, Rosner B, Stampfer MJ (1999) Age at natural menopause and risk of cardiovascular disease. Arch Intern Med 159:1061-1066

Hurt LS, Ronsmans C, Thomas SL (2006) The effect of number of births on women's mortality: systematic review of the evidence for women who have completed their childbearing. Popul Stud Camb 60:55-71

Kirkwood TB, Rose MR (1991) Evolution of senescence: late survival sacrificed for reproduction. Philos Trans R Soc Lond B Biol Sci 332:15-24

Kirkwood TBL (1977) Evolution of aging. Nature 270:301-304

Korpelainen H (2000) Fitness, reproduction and longevity among European aristocratic and rural Finnish families in the 1700s and 1800s. Proc Biol Sci 267:1765-1770

Kosova G, Abney M, Ober C (2010) Colloquium papers: heritability of reproductive fitness traits in a human population. Proc Natl Acad Sci USA 107 Suppl 1:1772-1778

Lawlor DA, Emberson JR, Ebrahim S, Whincup PH, Wannamethee SG, Walker M, Smith GD, British Women's Heart and Health Study, British Regional Heart Study (2003) Is the association between parity and coronary heart disease due to biological effects of pregnancy or adverse lifestyle risk factors associated with child-rearing? Findings from the British Women's Heart and Health Study and the British Regional Heart Study. Circulation 107:1260-1264

Le Bourg E (2007) Does reproduction decrease longevity in human beings? Ageing Res Rev 6:141-149

Lycett JE, Dunbar RI, Voland E (2000) Longevity and the costs of reproduction in a historical human population. Proc Biol Sci 267:31-35

Martin U, Davies C, Hayavi S, Hartland A, Dunne F (1999) Is normal pregnancy atherogenic? Clin Sci Lond 96:421-425

McArdle PF, Pollin TI, O'Connell JR, Sorkin JD, Agarwala R, Schaffer AA, Streeten EA, King TM, Shuldiner AR, Mitchell BD (2006) Does having children extend life span? A genealogical study of parity and longevity in the Amish. J Gerontol Biol Sci Med Sci 61:190-195

Muller HG, Chiou JM, Carey JR, Wang JL (2002) Fertility and life span: late children enhance female longevity. J Gerontol Biol Sci Med Sci 57:B202-B206

Nagirnaja L, Rull K, Uuskula L, Hallast P, Grigorova M, Laan M (2010) Genomics and genetics of gonadotropin betasubunit genes: unique FSHB and duplicated LHB/CGB loci. Mol Cell Endocrinol 329(1-2):4-16
Nakayama T, Kuroi N, Sano M, Tabara Y, Katsuya T, Ogihara T, Makita Y, Hata A, Yamada M, Takahashi N, Hirawa N, Umemura S, Miki T, Soma M (2006) Mutation of the follicle-stimulating hormone receptor gene 5'-untranslated region associated with female hypertension. Hypertension 48:512-518

Parazzini F, Negri E, La Vecchia C (1992) Reproductive and general lifestyle determinants of age at menopause. Maturitas 15:141-149

Penn DJ, Smith KR (2007) Differential fitness costs of reproduction between the sexes. Proc Natl Acad Sci USA 104:553-558

Pettay JE, Kruuk LE, Jokela J, Lummaa V (2005) Heritability and genetic constraints of life-history trait evolution in preindustrial humans. Proc Natl Acad Sci USA 102:2838-2843

Purcell S, Neale B, Todd-Brown K, Thomas L, Ferreira MA, Bender D, Maller J, Sklar P, de Bakker PI, Daly MJ, Sham PC (2007) PLINK: a tool set for whole-genome association and populationbased linkage analyses. Am J Hum Genet 81:559-575

Qureshi AI, Giles WH, Croft JB, Stern BJ (1997) Number of pregnancies and risk for stroke and stroke subtypes. Arch Neurol 54:203-206

Rendina D, Gianfrancesco F, De Filippo G, Merlotti D, Esposito T, Mingione A, Nuti R, Strazzullo P, Mossetti G, Gennari L (2010) FSHR gene polymorphisms influence bone mineral density and bone turnover in postmenopausal women. Eur J Endocrinol 163:165-172

Rose M, Charlesworth B (1980) A test of evolutionary theories of senescence. Nature 287:141-142

Sandler DP, Wilcox AJ, Horney LF (1984) Age at menarche and subsequent reproductive events. Am J Epidemiol 119:765-774

Sattar N, Greer IA (2002) Pregnancy complications and maternal cardiovascular risk: opportunities for intervention and screening? BMJ 325:157-160

Sowers MR, La Pietra MT (1995) Menopause: its epidemiology and potential association with chronic diseases. Epidemiol Rev 17:287-302

Stanford JL, Hartge P, Brinton LA, Hoover RN, Brookmeyer R (1987) Factors influencing the age at natural menopause. J Chronic Dis 40:995-1002

Stearns SC, Partridge L (2001) The genetics of aging in Drosophila. In: Masoro EJ, Austad SN (eds) Handbook of the biology of aging, 5th edn. Academic Press, Orlando, pp 345-360

Westendorp RG, Kirkwood TB (1998) Human longevity at the cost of reproductive success. Nature 396:743-746

Whelan EA, Sandler DP, McConnaughey DR, Weinberg CR (1990) Menstrual and reproductive characteristics and age at natural menopause. Am J Epidemiol 131:625-632

Williams GC (1957) Pleiotropy, natural selection, and the evolution of senescence. Evolution 11:398-411

Winkvist A, Rasmussen KM, Habicht JP (1992) A new definition of maternal depletion syndrome. Am J Public Health 82:691-694

Yang CQ, Chan KY, Ngan HY, Khoo US, Chiu PM, Chan QK, Xue WC, Cheung AN (2006) Single nucleotide polymorphisms of follicle-stimulating hormone receptor are associated with ovarian cancer susceptibility. Carcinogenesis 27:1502-1506 